\title{
Effects of Corneal Nerve Density on the Response to Treatment in Dry Eye Disease
}

\section{Citation}

Kheirkhah, Ahmad, Thomas H. Dohlman, Francisco Amparo, Michael A. Arnoldner, Arsia Jamali, Pedram Hamrah, and Reza Dana. 2015. "Effects of Corneal Nerve Density on the Response to Treatment in Dry Eye Disease." Ophthalmology 122 (4) (April): 662-668. doi:10.1016/ j.ophtha.2014.11.006.

\section{Published Version}

10.1016/j.ophtha.2014.11.006

\section{Permanent link}

http://nrs.harvard.edu/urn-3:HUL.InstRepos:34854275

\section{Terms of Use}

This article was downloaded from Harvard University's DASH repository, and is made available under the terms and conditions applicable to Open Access Policy Articles, as set forth at http:// nrs.harvard.edu/urn-3:HUL.InstRepos:dash.current.terms-of-use\#OAP

\section{Share Your Story}

The Harvard community has made this article openly available.

Please share how this access benefits you. Submit a story.

\section{Accessibility}




\title{
Effects of Corneal Nerve Density on the Response to Treatment in Dry Eye Disease
}

\author{
Ahmad Kheirkhah, MD, Thomas H Dohlman, MD, Francisco Amparo, MD, Michael A \\ Arnoldner, MD, Arsia Jamali, MD, MPH, Pedram Hamrah, MD, and Reza Dana, MD, MPH, \\ MSc \\ Cornea \& Refractive Surgery Service, Massachusetts Eye and Ear Infirmary, Department of \\ Ophthalmology, Harvard Medical School, Boston, MA, USA
}

\begin{abstract}
Purpose-To evaluate whether levels of corneal subbasal nerve fiber length (SNFL) in dry eye disease (DED) could prognosticate the level of improvement in signs and symptoms after treatment.

Design—Phase IV, double-masked, randomized clinical trial.

Participants-Sixty patients with meibomian gland dysfunction-associated DED and 27 agematched controls.

Methods-Patients with DED were randomized to receive topical artificial tears, loteprednol etabonate $0.5 \%$, or loteprednol etabonate $0.5 \%$ /tobramycin $0.3 \%$ twice daily for 4 weeks. At baseline, in vivo confocal microscopy of central cornea was performed in both eyes. Patients with DED were divided into 2 subgroups, those with low baseline SNFL and those with near-normal baseline SNFL (the cut-off point: mean SNFL in controls minus 2 standard deviations). Clinical signs and symptoms at baseline and after 4 weeks of treatment were compared between the subgroups with low and near-normal SNFL for all therapeutic groups.
\end{abstract}

Main Outcome Measures-Symptom questionnaires, corneal fluorescein staining (CFS), conjunctival staining with lissamine green, tear break-up time, Schirmer's test, and SNFL.

Results-In patients with DED, baseline SNFL $\left(17.06 \pm 5.78 \mathrm{~mm} / \mathrm{mm}^{2}\right)$ was significantly lower than in controls $(23.68 \pm 3.42, \mathrm{P}=0.001)$. In the artificial tear and loteprednol groups, although no significant improvement in any sign or symptom was noted in patients with low baseline SNFL $\left(<16.84 \mathrm{~mm} / \mathrm{mm}^{2}\right)$, subjects with near-normal baseline SNFL $\left(\geq 16.84 \mathrm{~mm} / \mathrm{mm}^{2}\right)$ showed

(C) 2014 by the American Academy of Ophthalmology. All rights reserved.

Corresponding Author: Reza Dana, MD, MPH, MSc, Massachusetts Eye and Ear Infirmary, 243 Charles Street, Boston, MA 02114, USA, Tel +1 617573 4439, Fax +1 617573 4300, Reza_Dana@meei.harvard.edu.

Meeting Presentation: This study has partly been presented at the Annual Meeting of the Association for Research in Vision and Ophthalmology, Orlando, FL, May 2014. This manuscript is not an Annual Meeting paper or poster.

Conflict of Interest: no conflicting relationship exists for any author.

Publisher's Disclaimer: This is a PDF file of an unedited manuscript that has been accepted for publication. As a service to our customers we are providing this early version of the manuscript. The manuscript will undergo copyediting, typesetting, and review of the resulting proof before it is published in its final citable form. Please note that during the production process errors may be discovered which could affect the content, and all legal disclaimers that apply to the journal pertain. 
significant improvement in both symptoms and corneal fluorescein staining (CFS) score (all $\mathrm{P}<0.05$ ). In the loteprednol/tobramycin group, no significant change was evident for any sign or symptom in either subgroup of low or near-normal baseline SNFL.

Conclusions-Significant improvements in CFS and patient symptomatology after DED treatment were evident only in the subgroup with near-normal corneal SNFL. Consideration of SNFL may thus assist in explaining the variability of patients' response to DED therapy.

\section{INTRODUCTION}

Dry eye disease (DED) is one of the most commonly encountered conditions in ophthalmic practice. It is estimated that over 5-30\% of the population aged 50 years or older suffer from DED, with a higher prevalence in some regions of the world, such as Asia. ${ }^{1-3}$ Although various subtypes of DED exist, such as aqueous-tear deficient and evaporative subtypes, the common denominator of the disease is tear film instability and ocular surface inflammation. ${ }^{4,5}$ This fact has clearly been reflected in the new definition of DED by Dry Eye WorkShop in 2007. ${ }^{6}$ Due to role of inflammation in the pathogenesis of DED, antiinflammatory agents are commonly used for treatment of DED, ${ }^{7}$ though with variable degrees of success. ${ }^{8,9}$ However, it remains unknown why not all patients with DED respond favorably to anti-inflammatory therapy.

The cornea is the most densely innervated tissue in the body with a nerve density of 300600 times that of the skin. ${ }^{10,11} \mathrm{~A}$ large number of studies have demonstrated that in addition to providing sensation, corneal nerves play a significant role in the maintenance of corneal epithelial health. ${ }^{11}$ These nerves, which promote epithelial proliferation and viability, ${ }^{12}$ have been shown to be reduced in a variety of ocular and systemic conditions, resulting in compromised ocular surface and reduced tear function. ${ }^{13,14}$ Examples include corneal insults such as infections, injuries, or surgeries, systemic disease like diabetes mellitus, and any damage to the trigeminal nerve. ${ }^{11,13,14}$ In addition to these conditions, the density of corneal nerves has been shown to be reduced in DED, ${ }^{15-22}$ which correlates with ocular surface staining. ${ }^{22}$ However, it remains unclear whether the density of corneal nerves at the time of treatment initiation plays any role in the therapeutic response in patients with DED.

As corneal nerves are required for the maintenance of ocular surface health, ${ }^{11,12}$ their trophic function may be important not only in normal conditions but also in disease states. Therefore, in this study we hypothesized that the response to treatment in patients with DED is dependent on the presence of near-normal corneal nerve density, and thus variable responses to DED therapy stem from different levels of corneal nerve density in each individual.

\section{MATERIALS AND METHOD}

\section{Study Design and Participants}

This prospective, double-masked, Phase IV randomized clinical trial included 60 patients with DED associated with meibomian gland dysfunction (MGD) who received one of the following medications for the treatment of ocular surface inflammation: loteprednol etabonate $0.5 \%$ ophthalmic suspension (Lotemax, Bausch \& Lomb Inc., Rochester, NY), a 
combination of loteprednol etabonate $0.5 \%$ and tobramycin $0.3 \%$ ophthalmic suspension (Zylet, Bausch \& Lomb Inc.), or artificial tear (Advanced Eye Relief® Dry Eye Environmental Lubricant Eye Drops, Bausch \& Lomb Inc.). The study protocol was approved by the Human Studies Committee of the Massachusetts Eye and Ear Infirmary, Boston, MA, and the research was conducted in accord with the requirements of the Health Insurance Portability and Accountability Act (HIPAA) and the tenets of the Declaration of Helsinki. This study was registered on clinicaltrials.gov (identifier NCT01456780).

This study included adult patients with MGD-associated DED. All patients had symptoms of DED with an Ocular Surface Disease Index (OSDI) score greater than 22, corneal fluorescein staining of 4 or more (National Eye Institute [NEI] grading scale, 0-15), and a diagnosis of MGD. The latter was diagnosed based on a systematic evaluation of the lid margin for obstruction of meibomian glands. Exclusion criteria consisted of the followings: use of steroids, antibiotics, or optical soft contact lenses within 2 weeks prior to enrollment; any change in the dosage of topical anti-inflammatory medications, other than steroids, or oral tetracyclines within 2 weeks prior to enrollment; and the use of isotretinoin within the past 6 months. Additional exclusion criteria included history of Stevens-Johnson syndrome or mucous membrane pemphigoid, history of herpetic keratitis, active ocular allergies, and allergy to aminoglycosides, steroids, or benzalkonium chloride. Potential participants were also excluded if they had a known history of glaucoma, an intraocular pressure $>22 \mathrm{mmHg}$ in either eye, or a known family history of glaucoma in a first degree relative.

Details of the study as well as the potential benefits and harms were thoroughly explained for the patients and all signed an informed consent form prior to participation in the study.

\section{Study Treatment}

These 60 patients were randomized to receive either loteprednol etabonate $0.5 \%$ ophthalmic suspension (Lotemax, $\mathrm{n}=20$ ), a combination of loteprednol etabonate $0.5 \%$ and tobramycin $0.3 \%$ ophthalmic suspension (Zylet, $\mathrm{n}=20$ ), or artificial tear (Advanced Eye Relief® Dry Eye Environmental Lubricant Eye Drops, n=20). All these medications were used twice daily for both eyes for 4 weeks, after which the study medication was discontinued.

\section{Clinical Evaluation}

Each participant had 3 clinic visits: before starting the study medication (baseline), after 4 weeks (range, \pm 7 days) of treatment, and 4 weeks (range, \pm 10 days) after discontinuation of the treatment ( 8 weeks after enrollment into the study). During each visit, all participants had a complete masked ophthalmic evaluation, which included the following: assessment of symptoms using OSDI as well as Symptom Assessment in Dry Eye (SANDE) questionnaires; measurement of the best corrected visual acuity and intraocular pressure; slit-lamp biomicroscopy to assess corneal fluorescein staining (NEI scale, 0-15), conjunctival staining with lissamine green (NEI scale, $0-18$ ), tear break-up time (TBUT), and Schirmer's test with anesthesia. 


\section{In Vivo Confocal Microscopy}

In this study, in vivo confocal microscopy (IVCM) was used to measure the corneal subbasal nerve fiber length (SNFL) before starting the treatment as well as after 4 weeks of therapy. All participants underwent laser IVCM of the central cornea in both eyes using Heidelberg Retina Tomograph 3 with the Rostock Cornea Module (Heidelberg Engineering, Heidelberg, Germany), as described before. ${ }^{23}$ This IVCM machine, which employs a 670-nm red wavelength diode laser, provides a magnification of 800 times and a lateral resolution of 1 $\mu \mathrm{m}$. It obtains digital images at a rate of 3 frames per second, with 100 images per sequence. Each image represents a coronal section of $384 \times 384$ pixels which is equivalent to $400 \times$ $400 \mu \mathrm{m}$ of the cornea. A total of three to five sequence scans were obtained from the fullthickness of the central cornea of eye, with at least 2 sequence scans focused on the subepithelial area and the subbasal nerve plexus, usually at a depth of 50-80 $\mu \mathrm{m}$. The images from subepithelial layer of the cornea were utilized to measure SNFL. For each eye, three most representative images of the subepithelial layer were chosen for the analysis.

To measure SNFL, the subbasal nerve fibers were traced using NeuronJ (http:// www.imagescience.org/meijering/software/neuronj/), which is a semi-automated nerve analysis plug-in of ImageJ (National Institutes of Health, USA). The SNFL was defined as the total length of all nerve fibers traced per a $0.16-\mathrm{mm}^{2}$ image, which was then expressed as $\mathrm{mm} / \mathrm{mm}^{2}$. To avoid subjective bias, two masked observers measured SNFL independently. The mean value of both observers was calculated, and for each patient the average of the SNFL values of both eyes was used for further analysis.

To compare the results of IVCM in DED patients to a normal group, the data from 27 agematched normal controls were used who did not have any sign or symptoms of DED. The SNFL measurement was done in the central cornea of both eyes of these individuals using IVCM, as described above. The SNFL measurements in the control group were used to define a cut-off point for having a low or near-normal SNFL. For this purpose, the cut-off point was considered as the mean value of SNFL in controls minus two standard deviations. Based on this cut-off point, DED patients in each of 3 treatment groups were divided into 2 subgroups, those with low baseline SNFL and those with near-normal baseline SNFL.

\section{Statistical Analysis}

Statistical analysis was performed with SPSS for Windows version 21 (SPSS Inc., Chicago, IL). For each variable, except for the symptoms, patient's data were calculated by averaging the scores from both eyes. The differences in each variable among the 3 treatment groups were compared with chi-square for the qualitative variables and the analysis of variance (ANOVA) with Bonferroni correction for quantitative variables. Repeated measure ANOVA was also used to compare the changes in each quantitative variable during 8 weeks of the study within each group. The differences between the subgroups of near-normal or low baseline SNFL were analyzed with independent sample $t$ test. Data normality was verified using Shapiro-Wilk test. Two-sided P value of less than 0.05 was considered as statistically significant for all comparisons. 


\section{RESULTS}

This study included 60 patients with MGD-associated DED who were equally randomized into 3 treatment groups. Out of these, 6 patients were either withdrawn or lost to follow-up prior to the 4-week visit. Furthermore, 3 additional subjects, one in each group, did not complete the 8-week visit. Therefore, 54 patients completed 4-week visit and enrolled in the analysis with 20 in the artificial tear group, 17 in the loteprednol etabonate group, and 17 in the loteprednol etabonate/tobramycin group. As has been demonstrated in Table 1, there were no significant differences among the three groups in terms of age, gender, and dry eye signs or symptoms, except for SANDE frequency which was higher in the loteprednol etabonate/tobramycin group.

Although there was no significant difference in SNFL among the 3 treatment groups (Table 1), SNFL was significantly lower in patients with DED $\left(17.06 \pm 5.78 \mathrm{~mm} / \mathrm{mm}^{2}\right)$ compared to the age-matched control group $\left(23.68 \pm 3.42 \mathrm{~mm} / \mathrm{mm}^{2}, \mathrm{P}=0.001\right)$. The cut-off point of $16.84 \mathrm{~mm} / \mathrm{mm}^{2}$ was then used to categorize the subjects with DED to those with low baseline SNFL $\left(<16.84 \mathrm{~mm} / \mathrm{mm}^{2}, \mathrm{n}=25\right)$ and those with near-normal baseline SNFL $(\geq 16.84$ $\left.\mathrm{mm} / \mathrm{mm}^{2}, \mathrm{n}=29\right)$.

The changes in signs and symptoms of DED after 4 weeks of treatment in each group have been presented in Tables 2-4. In the artificial tear group, although no significant improvement of any sign and symptom was noted in the subgroup with low baseline SNFL, there were significant improvements in the scores of SANDE frequency, SANDE severity, OSDI as well as corneal fluorescein staining in the subgroup with near-normal baseline SNFL (Table 2). In the subjects treated with loteprednol etabonate, the subgroup with low baseline SNFL did not show a significant improvement in any sign or symptom; however, those with near-normal baseline SNFL demonstrated significant improvements in SANDE severity and corneal fluorescein scores (Table 3). In the loteprednol etabonate/tobramycin group, no significant change was evident in any sign or symptom in either subgroups of low or near-normal baseline SNFL (Table 4).

Table 5 shows the changes in SNFL after 4 weeks of treatment in each group. Although the subjects in the artificial tear group did not demonstrate any significant change in mean SNFL, there were significant increases in mean SNFL in loteprednol etabonate and loteprednol etabonate/tobramycin groups ( $\mathrm{P}=0.04$ for both). During 4 weeks of follow-up after stopping the treatment, there were no significant changes in any sign or symptom in either subgroups of low or near-normal baseline SNFL in all treatment groups (Fig. 1).

\section{DISCUSSION}

This prospective, double-masked, randomized clinical trial showed that in subjects with MGD-associated DED who were treated with artificial tears or loteprednol etabonate, only subgroup with near-normal baseline SNFL demonstrated significant improvement in symptoms and corneal fluorescein staining. In contrast, there was no significant improvement after treatment in patients with low baseline SNFL. This SNFL-dependent differential response to DED treatment may, in part, explain the variability of patients' 
response to therapeutic modalities, which is commonly seen in clinical practice. If confirmed in additional larger studies, subject enrollment based on SNFL may assist in improving the predictability of response to therapy in future clinical trials for DED.

This study included 54 subjects with MGD-associated DED who also had a low mean Schirmer's test values (Table 1), implying the presence of mixed-mechanism DED with both MGD and aqueous tear deficiency playing a role in disease pathogenesis. Compared to an age-matched control group, the patients had significantly lower SNFL (23.68 \pm 3.42 versus $17.06 \pm 5.78 \mathrm{~mm} / \mathrm{mm}^{2}, \mathrm{P}=0.001$ ), as has been demonstrated before. ${ }^{15-22}$ Although morphologic changes in subbasal nerves, such as increased tortuosity, reflectivity, bead-like formation, have previously been reported in DED, ${ }^{15-22}$ these changes were not evaluated in this study, as there is no clear consensus on their quantification. On the other hand, an arbitrary cut-off point of mean minus 2 standard deviations of SNFL in the controls was used to categorize DED patients into those with low SNFL $\left(<16.84 \mathrm{~mm} / \mathrm{mm}^{2}\right)$ and those with near-normal SNFL ( $\geq 16.84 \mathrm{~mm} / \mathrm{mm}^{2}$ ).

Interestingly, in subjects treated with artificial tear or loteprednol etabonate, there was no statistically significant improvement in signs or symptoms after 4 weeks of treatment in the subgroups of low baseline SNFL (Tables 2 and 3). However, the subgroups with nearnormal baseline SNFL showed remarkable improvement in some symptom scores and corneal fluorescein staining, but no other DED signs. This may demonstrate a possible prognostic effect of corneal SNFL on the DED response to therapeutic modalities and thus may, in part, explain the individual variability in DED management trials.

Nerves have been shown to play a substantial role in the corneal epithelial homeostasis, ${ }^{11}$ and loss of corneal nerves has been associated with compromised corneal epithelial health, which is well exemplified in neurotrophic keratopathy. ${ }^{14,24}$ In DED, reduced corneal nerves compared to the controls have been reported in most studies, ${ }^{15-22}$ but not all. ${ }^{25-27} \mathrm{In}$ addition, corneal sensation has been demonstrated to be increased ${ }^{26,28}$ or decreased $^{16,29,30}$ in DED. These variations may be due to different subtypes of DED or different stages of the disease. ${ }^{31}$ We speculate that subjects with near-normal SNFL might have more functionally intact nerve endings, which may "sense" the improved ocular surface environment after the treatment, thus explaining the correlation between nerve density and improved symptoms. On the other hand, patients with severely damaged corneal nerves may not detect the posttreatment improvement in the ocular surface to the same degree. Improved corneal fluorescein staining after treatment in patients with near-normal baseline SNFL may also be attributed to the trophic support of the corneal nerves, which may help the renewal of a normal epithelium during the therapy. Future studies are certainly required to determine the mechanism by which higher baseline SNFL results in better outcomes of DED treatment.

Although the combination of loteprednol etabonate/tobramycin has been shown to be effective in treatment of blepharokeratoconjunctivitis, ${ }^{32-34}$ our study did not reveal any significant change in signs and symptoms of MGD-associated DED after 4 weeks of treatment with this combination in either subgroup of low or near-normal baseline SNFL (Table 4), which might, in part, be due to the potential toxicity of tobramycin component. ${ }^{35}$ Furthermore, in this study the individuals with near-normal baseline SNFL who were treated 
with loteprednol etabonate had improvement in fewer symptom scores than those who received artificial tears (Tables 2 and 3). Similarly, Pflugfelder and colleagues reported a significant reduction of symptoms after using loteprednol four times daily for 4 weeks, which was comparable to the placebo. ${ }^{8}$

At the IVCM level, our study revealed a significant increase in SNFL after treatment with loteprednol etabonate and loteprednol etabonate/tobramycin, but not in the artificial tear group (Table 5) despite the lack of clinical improvement in loteprednol/tobramycin group. It may thus be speculated that the anti-inflammatory regimen used in our study, although sufficient to induce cellular changes, was not sufficient to produce clinically noticeable improvement. Therefore, treatment with longer duration and/or higher dose of antiinflammatory medications may be necessary to achieve the clinical improvement in DED. ${ }^{36,37}$

In conclusion, our study revealed that response of DED to the treatment may be dependent on the individual patient's SNFL. Those with near-normal SNFL may have better response to DED therapy. This may help have a better predictability of patient's outcome in DED clinical trials.

\section{Acknowledgments}

Financial Support: Bausch \& Lomb, Inc., NIH K24-EY019098, Falk Medical Research Trust

\section{References}

1. The epidemiology of dry eye disease: report of the Epidemiology Subcommittee of the International Dry Eye WorkShop. Ocul Surf. 2007; 5:93-107. [PubMed: 17508117]

2. Lin PY, Tsai SY, Cheng CY, et al. Prevalence of dry eye among an elderly Chinese population in Taiwan: the Shihpai eye Study. Ophthalmology. 2003; 110:1096-101. [PubMed: 12799232]

3. Lee AJ, Lee J, Saw SM, et al. Prevalence and risk factors associated with dry eye symptoms: a population based study in Indonesia. Br J Ophthalmol. 2002; 86:1347-51. [PubMed: 12446361]

4. Pflugfelder SC, Jones D, Ji Z, et al. Altered cytokine balance in the tear fluid and conjunctiva of patients with Sjogren's syndrome keratoconjunctivitis sicca. Curr Eye Res. 1999; 19:201-11. [PubMed: 10487957]

5. Dana MR, Hamrah P. Role of immunity and inflammation in corneal and ocular surface disease associated with dry eye. Adv Exp Med Biol. 2002; 506(Pt B):729-38. [PubMed: 12613985]

6. The definition and classification of dry eye disease: report of the Definition and Classification Subcommittee of the International Dry Eye WorkShop. Ocul Surf. 2007; 5:75-92. [PubMed: 17508116]

7. Pflugfelder SC. Antiinflammatory therapy for dry eye. Am J Ophthalmol. 2004; 137:337-42. [PubMed: 14962426]

8. Pflugfelder SC, Maskin SL, Anderson B, et al. A randomized, double-masked, placebo-controlled, multicenter comparison of loteprednol etabonate ophthalmic suspension, $0.5 \%$, and placebo for treatment of keratoconjunctivitis sicca in patients with delayed tear clearance. Am J Ophthalmol. 2004; 138:444-57. [PubMed: 15364229]

9. Sall K, Stevenson OD, Mundorf TK, Reis BL. Two multicenter, randomized studies of the efficacy and safety of cyclosporine ophthalmic emulsion in moderate to severe dry eye disease. CsA Phase 3 Study Group. Ophthalmology. 2000; 107:631-9. [PubMed: 10768324]

10. Rózsa AJ, Beuerman RW. Density and organization of free nerve endings in the corneal epithelium of the rabbit. Pain. 1982; 14:105-20. [PubMed: 7177676] 
11. Müller LJ, Marfurt CF, Kruse F, Tervo TM. Corneal nerves: structure, contents and function. Exp Eye Res. 2003; 76:521-42. [PubMed: 12697417]

12. Marfurt CF, Cox J, Deek S, Dvorscak L. Anatomy of the human corneal innervation. Exp Eye Res. 2010; 90:478-92. [PubMed: 20036654]

13. Davis EA, Dohlman CH. Neurotrophic keratitis. Int Ophthalmol Clin. 2001; 41:1-11. [PubMed: 11198136]

14. Bonini S, Rama P, Olzi D, Lambiase A. Neurotrophic keratitis. Eye (Lond). 2003; 17:989-95. [PubMed: 14631406]

15. Benítez del Castillo JM, Wasfy MA, et al. An in vivo confocal masked study on corneal epithelium and subbasal nerves in patients with dry eye. Invest Ophthalmol Vis Sci. 2004; 45:3030-5. [PubMed: 15326117]

16. Benítez-Del-Castillo JM, Acosta MC, Wassfi MA, et al. Relation between corneal innervation with confocal microscopy and corneal sensitivity with noncontact esthesiometry in patients with dry eye. Invest Ophthalmol Vis Sci. 2007; 48:173-81. [PubMed: 17197530]

17. Erdélyi B, Kraak R, Zhivov A, et al. In vivo confocal laser scanning microscopy of the cornea in dry eye. Graefes Arch Clin Exp Ophthalmol. 2007; 245:39-44. [PubMed: 16874525]

18. Villani E, Galimberti D, Viola F, et al. The cornea in Sjogren's syndrome: an in vivo confocal study. Invest Ophthalmol Vis Sci. 2007; 48:2017-22. [PubMed: 17460255]

19. Villani E, Galimberti D, Viola F, et al. Corneal involvement in rheumatoid arthritis: an in vivo confocal study. Invest Ophthalmol Vis Sci. 2008; 49:560-4. [PubMed: 18234999]

20. Zhang X, Chen Q, Chen W, et al. Tear dynamics and corneal confocal microscopy of subjects with mild self-reported office dry eye. Ophthalmology. 2011; 118:902-7. [PubMed: 21146227]

21. Villani E, Magnani F, Viola F, et al. In vivo confocal evaluation of the ocular surface morphofunctional unit in dry eye. Optom Vis Sci. 2013; 90:576-86. [PubMed: 23670123]

22. Labbé A, Liang Q, Wang Z, et al. Corneal nerve structure and function in patients with non-sjogren dry eye: clinical correlations. Invest Ophthalmol Vis Sci. 2013; 54:5144-50. [PubMed: 23833066]

23. Cruzat A, Witkin D, Baniasadi N, et al. Inflammation and the nervous system: the connection in the cornea in patients with infectious keratitis. Invest Ophthalmol Vis Sci. 2011; 52:5136-43. [PubMed: 21460259]

24. Okada Y, Reinach PS, Kitano A, et al. Neurotrophic keratopathy; its pathophysiology and treatment. Histol Histopathol. 2010; 25:771-80. [PubMed: 20376784]

25. Tuominen IS, Konttinen YT, Vesaluoma MH, et al. Corneal innervation and morphology in primary Sjögren's syndrome. Invest Ophthalmol Vis Sci. 2003; 44:2545-9. [PubMed: 12766055]

26. Tuisku IS, Konttinen YT, Konttinen LM, Tervo TM. Alterations in corneal sensitivity and nerve morphology in patients with primary Sjögren's syndrome. Exp Eye Res. 2008; 86:879-85. [PubMed: 18436208]

27. Zhang M, Chen J, Luo L, et al. Altered corneal nerves in aqueous tear deficiency viewed by in vivo confocal microscopy. Cornea. 2005; 24:818-24. [PubMed: 16160498]

28. De Paiva CS, Pflugfelder SC. Corneal epitheliopathy of dry eye induces hyperesthesia to mechanical air jet stimulation. Am J Ophthalmol. 2004; 137:109-15. [PubMed: 14700652]

29. Xu KP, Yagi Y, Tsubota K. Decrease in corneal sensitivity and change in tear function in dry eye. Cornea. 1996; 15:235-9. [PubMed: 8713924]

30. Bourcier T, Acosta MC, Borderie V, et al. Decreased corneal sensitivity in patients with dry eye. Invest Ophthalmol Vis Sci. 2005; 46:2341-5. [PubMed: 15980220]

31. Alhatem A, Cavalcanti B, Hamrah P. In vivo confocal microscopy in dry eye disease and related conditions. Semin Ophthalmol. 2012; 27:138-48. [PubMed: 23163268]

32. Rhee SS, Mah FS. Comparison of tobramycin $0.3 \%$ /dexamethasone $0.1 \%$ and tobramycin $0.3 \%$ / loteprednol $0.5 \%$ in the management of blepharo-keratoconjunctivitis. Adv Ther. 2007; 24:60-7. [PubMed: 17526462]

33. White EM, Macy JI, Bateman KM, Comstock TL. Comparison of the safety and efficacy of loteprednol $0.5 \%$ /tobramycin $0.3 \%$ with dexamethasone $0.1 \%$ /tobramycin $0.3 \%$ in the treatment of blepharokeratoconjunctivitis. Curr Med Res Opin. 2008; 24:287-96. [PubMed: 18062846] 
34. Chen M, Gong L, Sun X, et al. A multicenter, randomized, parallel-group, clinical trial comparing the safety and efficacy of loteprednol etabonate $0.5 \%$ /tobramycin $0.3 \%$ with dexamethasone $0.1 \%$ / tobramycin $0.3 \%$ in the treatment of Chinese patients with blepharokeratoconjunctivitis. Curr Med Res Opin. 2012; 28:385-94. [PubMed: 22256909]

35. Lass JH, Mack RJ, Imperia PS, et al. An in vitro analysis of aminoglycoside corneal epithelial toxicity. Curr Eye Res. 1989; 8:299-304. [PubMed: 2707046]

36. Hong S, Kim T, Chung SH, et al. Recurrence after topical nonpreserved methylprednisolone therapy for keratoconjunctivitis sicca in Sjögren's syndrome. J Ocul Pharmacol Ther. 2007; 23:78-82. [PubMed: 17341155]

37. Wan PX, Wang XR, Song YY, et al. Study on the treatment of dry eye with Loteprednol Etabonate. Zhonghua Yan Ke Za Zhi. 2012; 48:142-7. [PubMed: 22490950] 


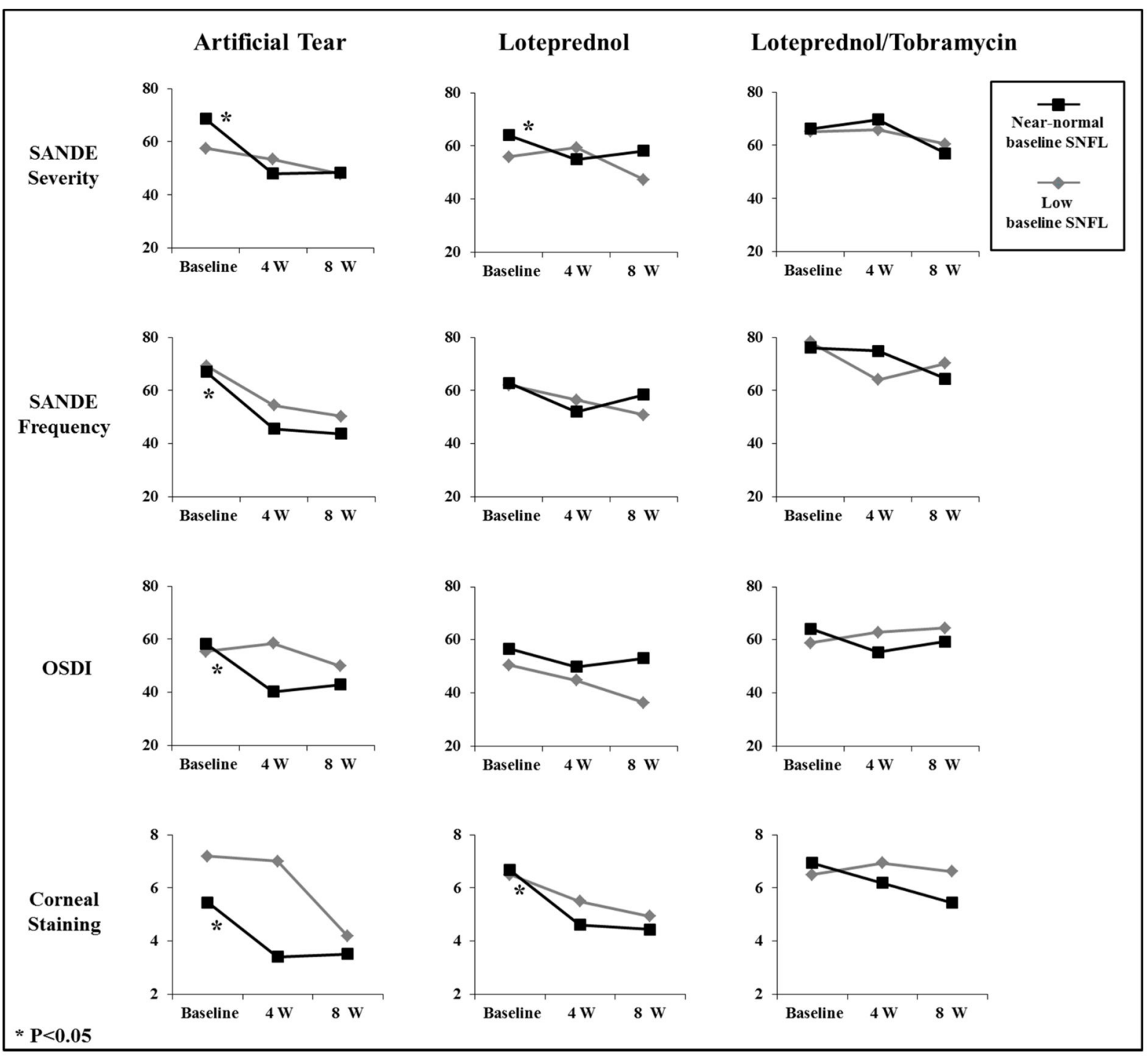

Fig. 1.

Changes in symptoms and signs in the subgroups with low or near-normal baseline subbasal nerve fiber length (SNFL) after 4 weeks of treatment with artificial tears, loteprednol etabonate, and loteprednol etabonate/tobramycin as well as during 4 weeks after stopping the treatment. 


\section{Table 1}

Clinical characteristics and subbasal nerve fiber length (SNFL) in all treatment groups at the baseline.

\begin{tabular}{|l|c|c|c|c|}
\hline & Artificial Tear (n=20) & Loteprednol Etabonate (n=17) & Loteprednol Etabonate/Tobramycin (n=17) & P value \\
\hline Age (years) & $57 \pm 12$ & $52 \pm 12$ & $55 \pm 13$ & 0.48 \\
\hline Gender (F/M) & $15 / 5$ & $9 / 8$ & $8 / 9$ & 0.18 \\
\hline OSDI & $57.0 \pm 21.3$ & $52.0 \pm 22.6$ & $59.7 \pm 18.0$ & 0.55 \\
\hline SANDE Frequency & $68.1 \pm 17.8$ & $60.9 \pm 19.4$ & $77.8 \pm 14.2$ & 0.02 \\
\hline SANDE Severity & $64.2 \pm 19.5$ & $58.1 \pm 19.6$ & $67.0 \pm 19.8$ & 0.41 \\
\hline $\begin{array}{l}\text { Corneal Fluorescein } \\
\text { Staining (NEI scale) }\end{array}$ & $6.1 \pm 2.0$ & $6.6 \pm 2.5$ & $6.8 \pm 2.6$ & 0.67 \\
\hline $\begin{array}{l}\text { Tear Break-up Time } \\
\text { (seconds) }\end{array}$ & $3.7 \pm 2.1$ & $2.9 \pm 1.5$ & $2.8 \pm 2.2$ & 0.31 \\
\hline $\begin{array}{l}\text { Conjunctival } \\
\text { Lissamine Green } \\
\text { Staining (NEI scale) }\end{array}$ & $1.9 \pm 1.4$ & $3.3 \pm 3.7$ & $2.4 \pm 2.2$ & 0.23 \\
\hline Schirmer's Test (mm) & $4.8 \pm 3.7$ & & & $5.7 \pm 7.3$ \\
\hline $\begin{array}{l}\text { Subbasal Nerve Fiber } \\
\left.\text { Length (mm/mm }{ }^{2}\right)\end{array}$ & $18.4 \pm 4.8$ & $5.8 \pm 6.1$ & $17.1 \pm 5.5$ & 0.83 \\
\hline
\end{tabular}

F: Female, M: male. 


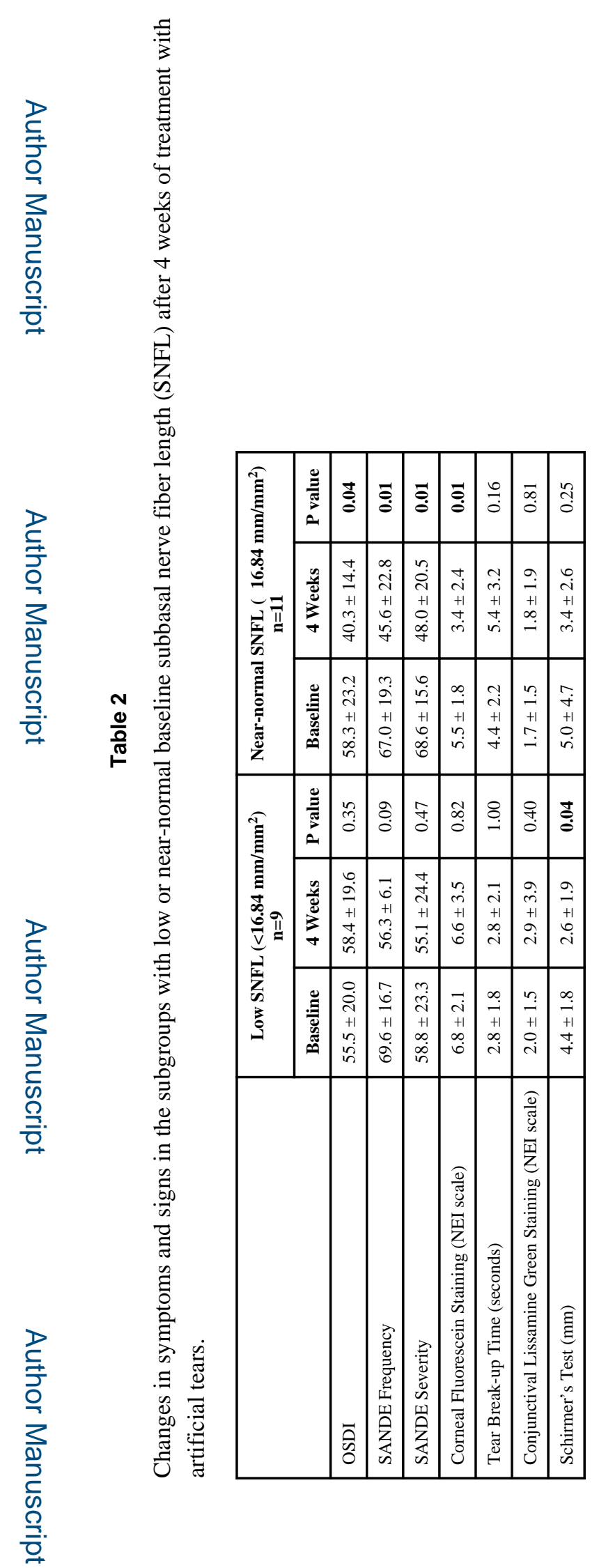

Ophthalmology. Author manuscript; available in PMC 2016 April 01. 


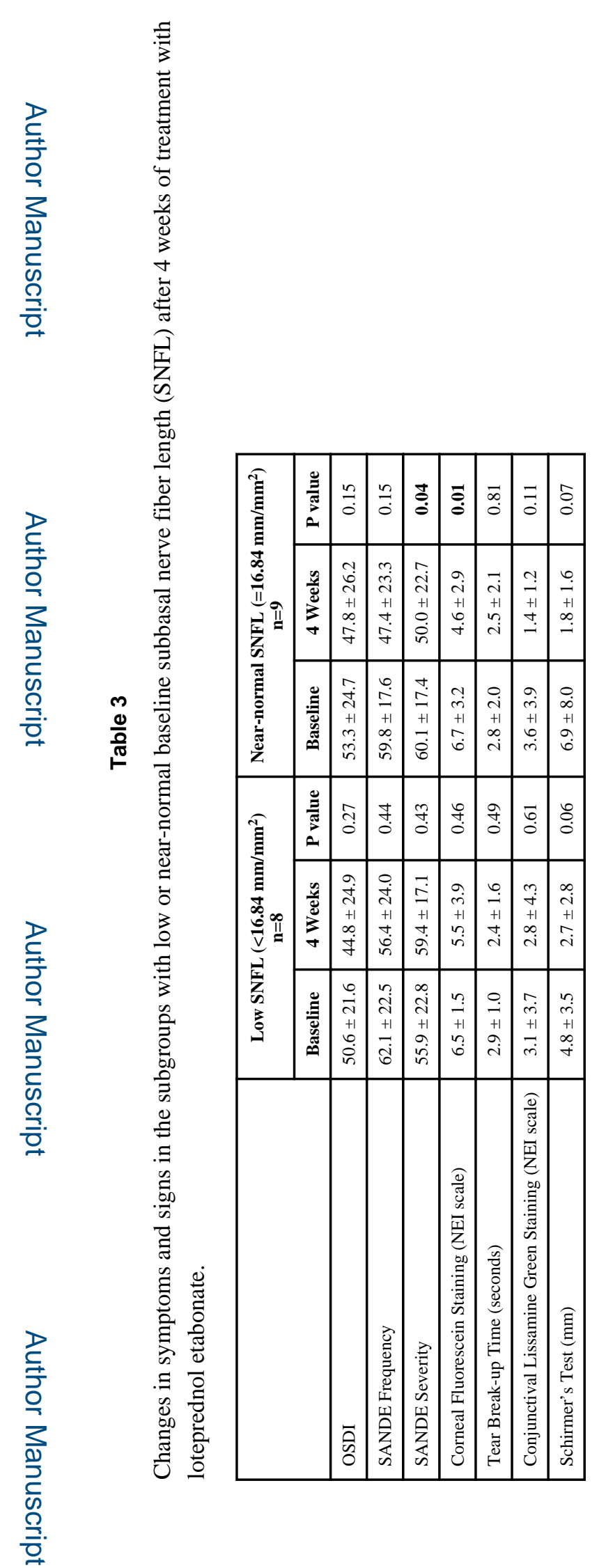

Ophthalmology. Author manuscript; available in PMC 2016 April 01. 


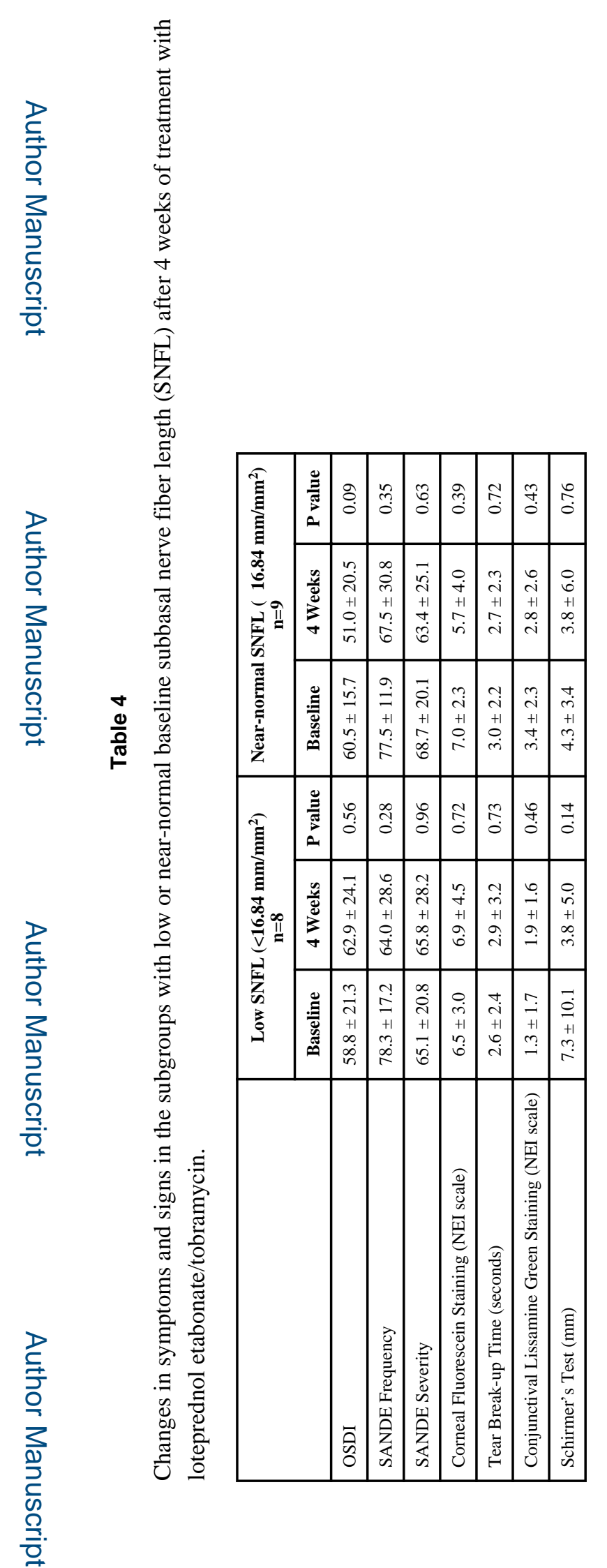

Ophthalmology. Author manuscript; available in PMC 2016 April 01. 
Table 5

Changes in subbasal nerve fiber length (SNFL) after 4 weeks of treatment in different groups.

\begin{tabular}{|l|c|c|c|}
\hline \multirow{2}{*}{} & \multicolumn{3}{|c|}{ Subbasal Nerve Fiber Length $\left(\mathbf{m m} / \mathbf{m m}^{\mathbf{2}}\right)$} \\
\cline { 2 - 4 } & Baseline & 4 Weeks & P value \\
\hline Artificial Tear & $18.4 \pm 4.8$ & $19.1 \pm 5.4$ & 0.23 \\
\hline Loteprednol Etabonate & $15.5 \pm 7.0$ & $17.4 \pm 5.2$ & 0.04 \\
\hline Loteprednol Etabonate/Tobramycin & $17.1 \pm 5.5$ & $19.1 \pm 8.2$ & 0.04 \\
\hline
\end{tabular}

